\title{
A modular, multi-diagnostic, automated shock tube for gas-phase chemistry
}

Cite as: Rev. Sci. Instrum. 90, 064104 (2019); https://doi.org/10.1063/1.5095077

Submitted: 07 March 2019 . Accepted: 01 June 2019 . Published Online: 21 June 2019

Mark E. Fuller (D), Mal Skowron, Robert S. Tranter (D), and C. Franklin Goldsmith (D)

\section{COLLECTIONS}

F This paper was selected as Featured
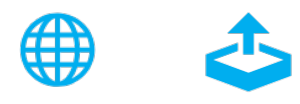

\section{ARTICLES YOU MAY BE INTERESTED IN}

High sensitivity microwave spectroscopy in a cryogenic buffer gas cell

Review of Scientific Instruments 90, 053104 (2019); https://doi.org/10.1063/1.5091773

Development of a compact tunable diode laser absorption spectroscopy based system for continuous measurements of dissolved carbon dioxide in seawater

Review of Scientific Instruments 90, 065110 (2019); https://doi.org/10.1063/1.5095797

A direct comparison of high-speed methods for the numerical Abel transform

Review of Scientific Instruments 90, 065115 (2019); https://doi.org/10.1063/1.5092635

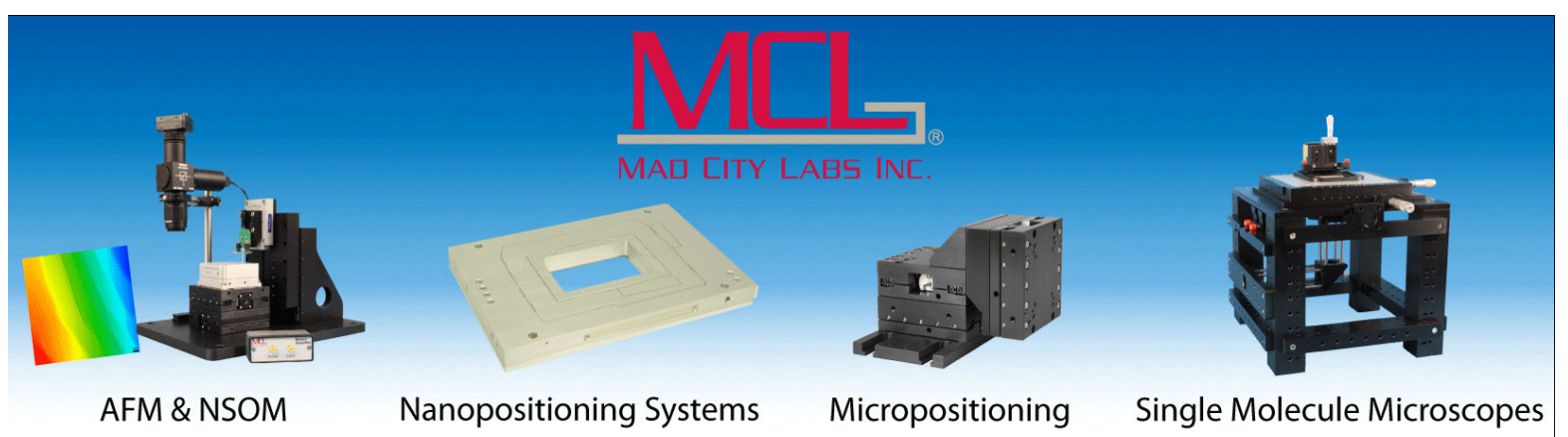




\title{
A modular, multi-diagnostic, automated shock tube for gas-phase chemistry
}

\author{
Cite as: Rev. Sci. Instrum. 90, 064104 (2019); doi: 10.1063/1.5095077 \\ Submitted: 7 March 2019 • Accepted: 1 June 2019 • \\ Published Online: 21 June 2019
}

\begin{abstract}
Mark E. Fuller, ${ }^{1}$ (D) Mal Skowron, ${ }^{1}$ Robert S. Tranter, ${ }^{2}$ (D) and C. Franklin Goldsmith ${ }^{1, a)}$ (D)
AFFILIATIONS

${ }^{1}$ School of Engineering, Brown University, 184 Hope Street, Providence, Rhode Island 02912, USA

${ }^{2}$ Chemical Sciences and Engineering Division, Argonne National Laboratory, Argonne, IIlinois 60439, USA
\end{abstract}

a) Author to whom correspondence should be addressed: franklin_goldsmith@brown.edu

\begin{abstract}
A new shock tube has been constructed for investigations of high-temperature chemical kinetics with an emphasis on combustion chemistry. This instrument includes a diaphragmless driver and electrical control of valving. A diaphragmless design significantly improves repeatability of experimental conditions vs the use of diaphragms and leads to an approximate order of magnitude reduction in turnaround time between experiments. Electrical control of valves, combined with diaphragmless operation, also enables remote and automated operation of the shock tube. The design allows for both incident and reflected shock experiments with multiple diagnostics. The performance of the shock tube is demonstrated by reproducing previous literature measurements on the unimolecular decomposition of isobutyl nitrite and cyclohexene.
\end{abstract}

Published under license by AIP Publishing. https://doi.org/10.1063/1.5095077

\section{INTRODUCTION}

A new experimental apparatus has been designed and constructed at Brown University. The new Brown Shock Tube (BST) has been developed for gas-phase chemical kinetic investigations over a broad range of temperatures and pressures $(\sim 700-2000 \mathrm{~K}$, 0.06-100 atm).

The BST is equipped with a diaphragmless driver section that gives excellent reproducibility of shock conditions. ${ }^{1,2}$ The diaphragmless driver also significantly reduces turnaround time between experiments, by about an order of magnitude. The BST is highly modular and can be easily reconfigured to implement multiple analytical techniques and types of experiments. The BST is currently configured for laser schlieren densitometry behind the incident shock wave, which is described in detail in Sec. II C. Future work will include optical diagnostics behind reflected shock waves, ignition delay measurements, and gas sampling.

The capabilities of this new apparatus are demonstrated by replication of previously measured decomposition rate constants for isobutyl nitrite ${ }^{3}$ and cyclohexene ${ }^{4}$ by laser-schlieren densitometry (LS). The LS technique is most suitable for low pressures $(<1 \mathrm{~atm})$ but tests the salient features of the BST. The present work shows excellent agreement within experimental error with the prior literature results, indicating that the apparatus is performing as intended.

\section{METHODS/EXPERIMENTAL}

\section{A. Shock tube design and construction}

The BST is a unique instrument in terms of the features in design, construction, modularity, and flexibility. The BST utilizes a diaphragmless driver modeled on the design currently in use at Argonne National Laboratory. ${ }^{2}$ The driver section is short in length and oversized in diameter relative to the driven section. At the start of an experiment, the driver and driven sections are isolated from each other with a bellows-actuated pneumatic valve instead of the more traditional disposable diaphragm. The diaphragmless driver section of the BST is shown in Fig. 1 and is similar to that in Ref. 2. The valving used to replace diaphragms and produce shock waves consists of a piston seal created by a plate with a circumferential O-ring which seals in a throat. The plate is mounted on a shaft which is linearly actuated using a metal bellows, confined within a pressure vessel ("can") inside the driver section. The shaft is supported with a linear bearing to ensure alignment and the bellows is backed with a neoprene bumper to prevent overexpansion. Tie rods to mount 


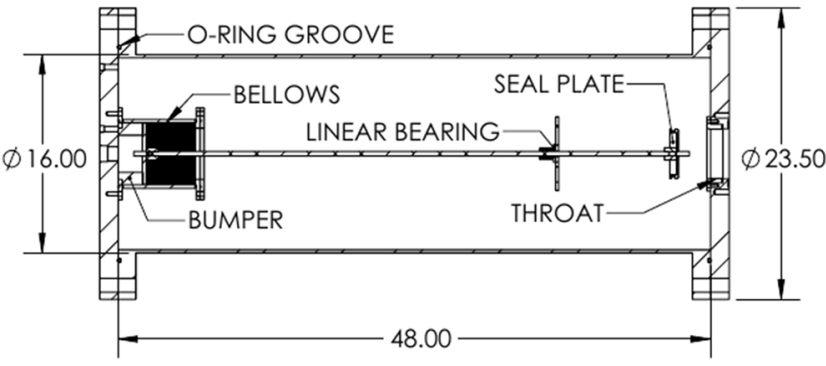

FIG. 1. Diaphragmless driver section cutaway view. Dimensions in inches.

and secure the can assembly and support rods for the linear bearing assembly are not shown for image clarity in Fig. 1. Details of the process by which the valve is actuated to produce shocks are described below in Sec. II B.

The driver section of the BST is $4 \mathrm{ft}$. $(1.2 \mathrm{~m})$ in length and is constructed from standard 16 in. nominal size ASME flanges and schedule 10 pipe. The interior of the driver section was polished to an ASTM A480/A480M \#3 finish (125 rms). The flanges were modified from stock to seal with O-rings instead of flat gaskets by cutting full-depth O-ring grooves on the flanges welded to the pipe. All flanges and pipes are made of 304 stainless steel. The driven section has an overall length of $24 \mathrm{ft} .(7.3 \mathrm{~m})$ and an inner diameter of $4.02 \mathrm{in}$. $(10.2 \mathrm{~cm})$. The driver-to-driven volume ratio is approximately 2.5 , with larger volume ratios allowing for stronger shocks for the same initial pressures in the driver and driven sections. The dimensions of the driven section provide a length-to-diameter (L/D) ratio of $\gtrsim 70$, which is typical of an optical shock tube. ${ }^{5}$ The length was selected as a compromise for the given laboratory space, gas consumption, and desired diagnostics. Boundary layer effects are mitigated with increased tube diameter: large diameter shock tubes $(D \gtrsim 10 \mathrm{~cm})$ have small boundary layers relative to the bore and are well approximated by normal shock equations.

Each piece of the driven section was constructed from a single billet of solid 304 stainless steel in order to avoid any variation caused by welding or asymmetries that could result from the extruded tube. The weldless construction of a shock tube from solid billets has been previously described by Tranter et al. ${ }^{6}$ Sections were machined by first boring out the interior and then turning back the stock to ensure concentricity before any additional features were machined. The bore diameter is precise to $\pm 0.002 \mathrm{in}$. The fabrication of the shock tube sections from solid billets results in design trade-offs vs construction from the tube or pipe and weldments. As shown in Fig. 2, rather than welding on a block of material to reinforce the window penetration and provide depth for threaded fasteners, the tube section was first bored to the desired inner diameter and then turned to provide a thickened section in the middle of the tube, reducing the amount of material to be removed and machining time. Window penetrations and mounting surfaces were then machined onto the thickened section and a single slot was milled for mounting of pressure transducers. The slot is necessary to mount all transducers on a consistent wall thickness with the same threaded interface, making the transducer hardware interchangeable. Additional tube sections for laser absorption spectroscopy and ignition

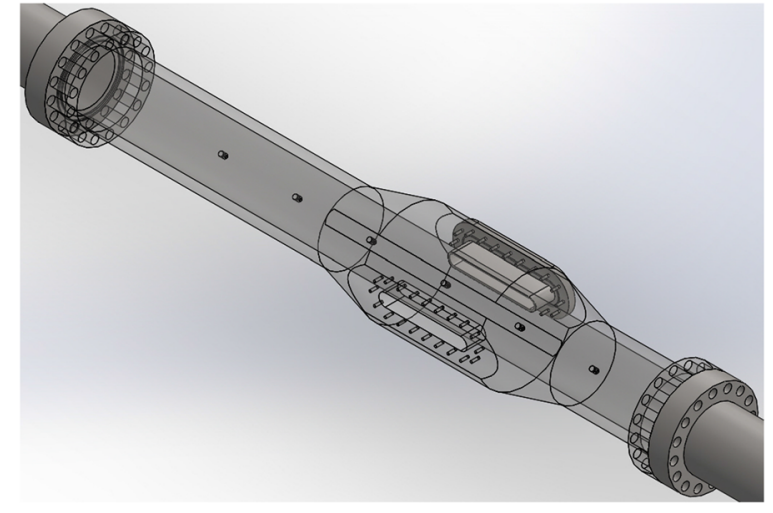

FIG. 2. Schlieren diagnostic section: the near-side penetration is open and a window frame is shown installed on the far-side. The six points correspond to the location of the pressure transducers.

delay, Figs. 3 and 4, have also been manufactured with the same transducer ports allowing transducers to be switched between sections and shock velocities to be measured close to the observation point in all BST configurations. The transducers are discussed below in Sec. II B.

Tube sections were designed according to the ASME Boiler and Pressure Vessel Code (BPVC) ${ }^{7}$ to obtain a working pressure of up to $100 \mathrm{~atm}$ at a factor of safety of at least two. To minimize weight and material costs, a custom flange pattern for joining the tube sections together was developed following the $\mathrm{BPVC}^{8}$ and ASME literature. ${ }^{9}$

The flange design requires 16 grade $51 / 2-13$ bolts to join the two flanges together. These flanges are symmetric, with each having a half-depth $\mathrm{O}$-ring groove and inner and outer backup rings to support the O-ring and aid in alignment during assembly. The backed-up O-ring seals are based upon the design developed in Ref. 6.

Window ports are incorporated in the driven section in two locations: As shown in Fig. 2, a long pair of windows, principally used for laser-schlieren measurements in the incident shock, are

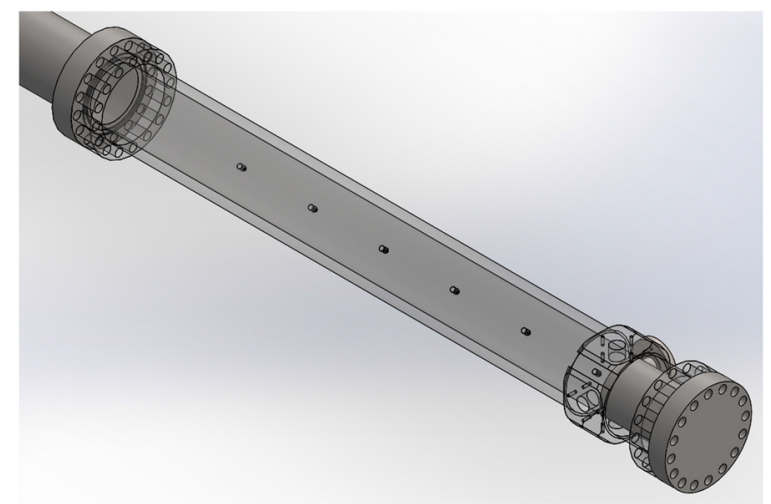

FIG. 3. Absorption diagnostic section. The six points are the sidewall pressure transducer ports. 

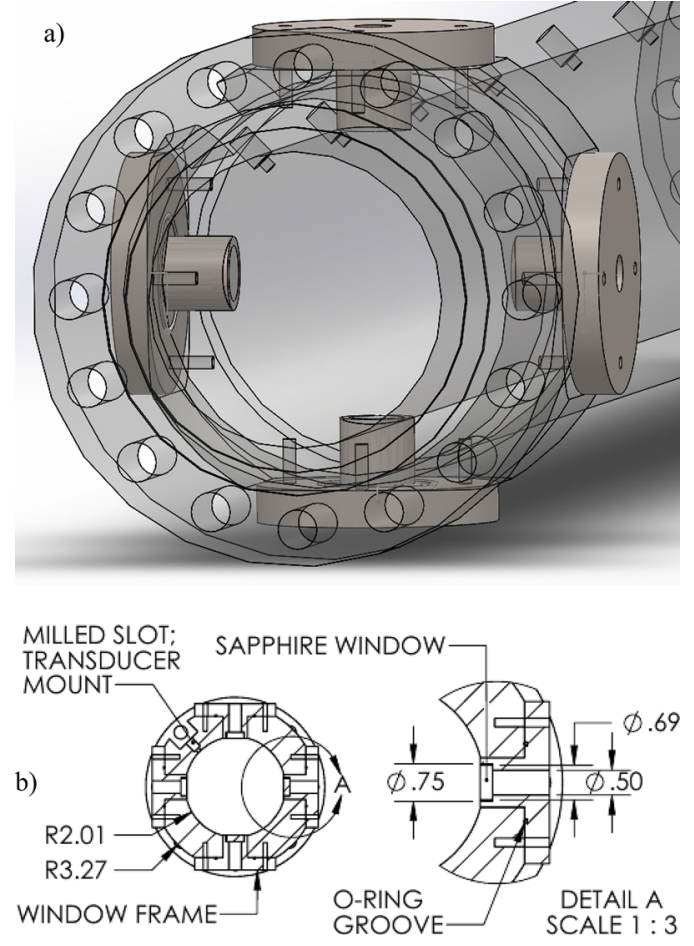

FIG. 4. Absorption window inserts. (a) 3D rendering. (b) Cutaway view showing the window detail in (a) and the milled slot for the pressure transducers. The cross-sectional profile shown of the window ports is common between the laser absorption and laser schlieren windows. Dimensions in inches.

located far from the end of the driven section. For experiments in the reflected shock, an additional set of windows are located close to the endwall of the driven section in a short removable section (Figs. 3 and 4). The two pairs of ports in Fig. 3 allow for multiple simultaneous diagnostics at the endwall. Sealing, mounting, and common dimensions for both sets of windows are provided in Fig. 4(b).

Window frames were designed iteratively by calculating screw loadings ${ }^{10}$ and window stress, ${ }^{11}$ and with finite element analysis (FEA) in the Solidworks computer-aided design software. ${ }^{12}$ Penetrations were designed and evaluated for the same working pressure of $100 \mathrm{~atm}$ as the main shock tube.

Although all window frames are designed for 100 atm, not all the window materials can withstand this pressure. Optimum window materials are selected for each diagnostic. However, all the current windows are rated for a minimum working pressure of 300 psi (gauge) ( 20 atm) and the current system is regulated and relieved to prevent loading beyond 150 psi (gauge). For experiments that do not require windows, they are either replaced with blank plates machined with the same profile as the window mounts or the entire tube section is replaced by one with no penetrations and mounts.

C-axis sapphire is used for the windows in Fig. 4, which are rated for $100 \mathrm{~atm}$ and intended for a single-pass laser absorption diagnostic, a technique described by Hanson and Davidson. ${ }^{5}$ The usable openings of the windows are $0.5 \mathrm{in}$. in diameter. A 0.69 in. diameter sapphire window is adhered in a 0.75 in. diameter recess within the frame (Fig. 4). The windows have a thickness of $0.25 \mathrm{in}$.

The windows shown in Fig. 2 are constructed from fused silica and are rated for 300 psi (gauge). Each window has a thickness of $0.5 \mathrm{in}$. The usable window area is a $0.5 \mathrm{in}$. diameter by $6 \mathrm{in}$. length slot; the windows have the shape of a 0.69 in. diameter, 6 in. slot and the window frames are milled out with a 0.75 in. by 6 in. slot to receive the window (Fig. 4). The choice of weaker fused silica over sapphire is due to sapphire's birefringence, which would interfere with the proper functioning of the laser-schlieren diagnostic, discussed below in Sec. II C. Furthermore, the LS experiments are typically limited to low pressures. ${ }^{13}$

The final stage of machining was to hone and polish the entire driven section interior to an ASTM A480/A480M \#8 mirror finish ( $<3 \mathrm{rms}$ ). Additionally, the driven section was treated with SilcoNert 2000, by SilcoTek, to minimize surface reactions and adsorption. The surface was polished and coated to reduce absorption of material on the wall and to reduce artifacts that might be introduced into the studies by a nonsmooth surface. The shock tube was tested to 1.3 times the operating range with pressurized water to verify its ability to safely hold pressure.

Two endwalls have been manufactured for use with different experimental configurations: A flat-cap endwall has been manufactured for use with laser-schlieren experiments, and a "top hat" plug endwall for laser-absorption or ignition delay measurements. The "top hat" configuration locates the position of the endwall $1 \mathrm{~cm}$ from the center of optical diagnostic window. The tube section for endwall diagnostics is shown in Fig. 3 with the top hat endwall installed. A closeup view of the mounting inserts in the tube penetrations is shown in Fig. 4.

A piston valve port for access to the driven section is adapted from the Petersen group at Texas A\&M. ${ }^{14}$ The tube side of the piston is cut to match the curvature of the wall, and the shaft is keyed to make it irrotational. In the closed position, the piston forms a flush and smooth wall to the shock tube other than the necessary diametrical clearance, a loose fit of $0.1 \mathrm{in}$. This valve is placed immediately downstream of the driver-driven interface at approximately three diameters of the driven section length (Fig. 5). The shaping of the piston and its location serve to minimize its impact on flow in the shock tube as full development of the shock requires approximately 20 diameters of tube length.

\section{B. Shock tube controls and operation}

For fill and fire operations, all valves are operated electronically from a software control panel. All solenoid valves and airsupply solenoids for pneumatic valves are controlled via a relay board with serial communication supplied by RelayPros LLC. Passing of setpoints to the proportional valves, toggling of relays to actuate two-way valves, and monitoring of pressure and temperature readouts is consolidated in the software control panel. The software panel was written using LabVIEW 2013 and provides a unified interface to drivers and subprograms for the relay board, pressure controllers, pressure transducers, Measurement Computing Corporation (MCC) thermocouple board, and MKS manometer and cold cathode pressure readouts.

The driver section and backing gas for the driver bellows are metered by two pressure controllers (Alicat), which have onboard 


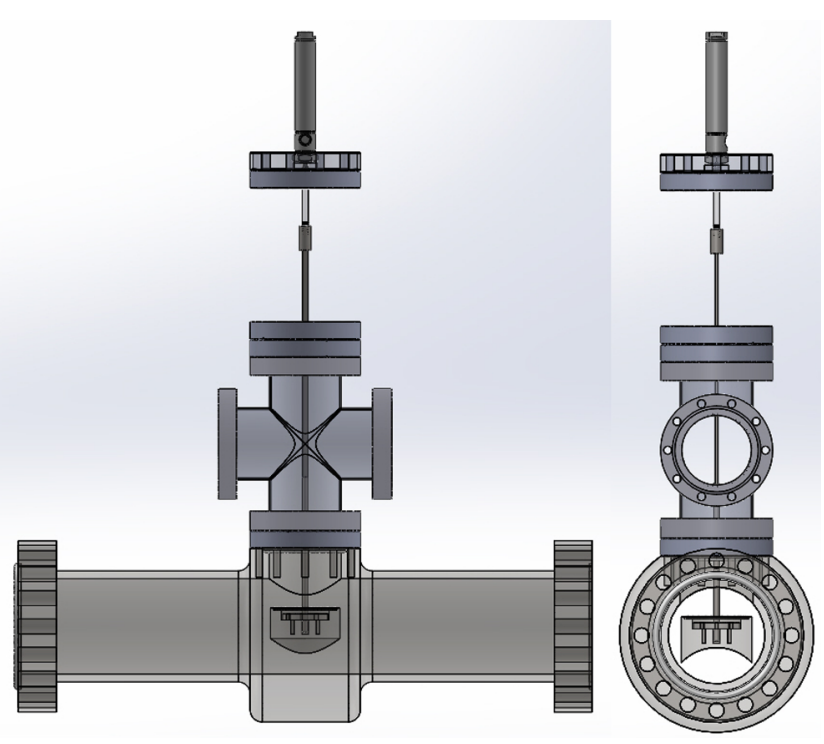

FIG. 5. Driven section piston valve.

proportional integral derivative (PID) control to fill to a given setpoint. The pressure controllers are identified as "PV," proportional valve, in Fig. 6. All Alicat pressure controllers have turndown of $200: 1$, meaning that they can precisely control to $0.5 \%$ of full scale.

Controller PV1 (Alicat PCS-500PSIA-D-PCA13) is set for the desired back pressure in the bellows, approximately 5 psi greater than the desired driver pressure and the internal pressure vessel in the driver ("can") is automatically filled to isolate the driver and driven sections.

A second controller, PV2 (Alicat PCRDS-500PSIA-D-20X32), is used to fill the driver section. Controller PV2 is a PCRDS series valve with exhaust and can correct for an overfill, ensuring that the desired pressure is achieved. Both controllers PV1 and PV2 are configured for 250 psi (absolute) full-scale operation. These controllers are not sufficient for producing reflected shocks at $P_{5}=100 \mathrm{~atm}$, but could be reconfigured or replaced with drop-in replacements from the same manufacturer. Current stock controllers are available to 3000 psi (absolute).

The remaining valves are designated as "BV," binary valve (2-way valves, either open or closed) and consist of both solenoid and pneumatic valves. To fire the shock tube, a pneumatic ball valve, BV0, is toggled open to release the gas in the can into the dump tank, "DT," which has previously been evacuated. The connection between the dump tank and rough pump, VP1, is controlled with solenoid valve BV1. Following the shock, solenoid valve BV2 is opened to evacuate the tube with pump VP1. Valves BV0, BV1, and BV2 were purchased from Bürkert: BV0 is a 8805 series ball-valve; BV1 and BV2 are series 0290 solenoid valves. All three valves are configured normally closed.

Additionally, the driver and can pressures are monitored with pressure transducers PT01 and PT02 (Omega Engineering MMA250USBHP6C0T8A9CE), 250 psi (absolute) full-scale range.

Remote filling of the driven section is accomplished with a third pressure controller (Alicat PCDS-100TORRA-D-PCA15). This controller, PV3, is a PCDS series controller with an exhaust valve, like PV2, and is compatible with a wide range of gas mixtures. The full-scale range of the controller is 100 Torr. For higher pressure experiments, an alternate controller with a full-scale range of 30 psi (absolute) (Alicat PCDS-30PSIA-D-PCV65) is also available.

For the experimental results presented here, filling of the driven section was accomplished manually, as controller PV3 had not yet been installed into the gas manifold.

Pressure during mixture preparation and filling of the driven section of the shock tube is monitored using a MKS 937B Gauge Controller connected to four Baritron manometers and a cold cathode ionization gauge. Manometers with full scale ranges of 1, 10, 100, and 1000 Torr (MKS 626 series) allow for measurement of atmospheric pressure down to below $1 \times 10^{-3}$ Torr. Below, $1 \times 10^{-3}$ Torr, the cold cathode (MKS series 423 ionization gauge) provides pressure readings.

The piston valve connecting the gas mixing manifold to the driven section is controlled with a commercial air-cushioned, dualacting air cylinder, which is controlled via a $4 / 2$ solenoid valve.

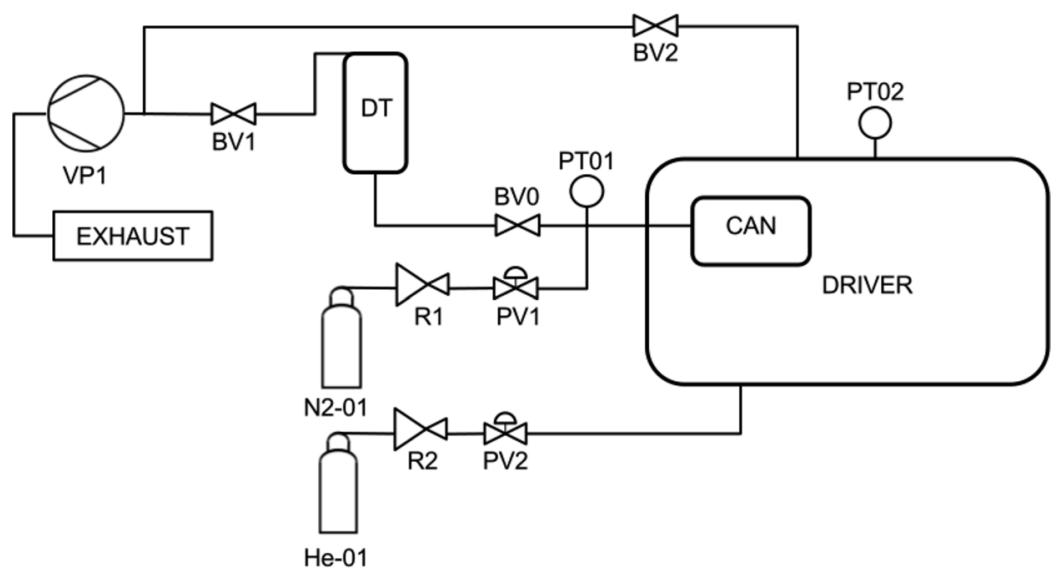

FIG. 6. BST driver schematic layout. 
The mixing manifold is connected to its own rough pump via a pneumatic angled bellows valve (Lesker). Temperature of the shock tube is monitored with a surface-mount type K thermocouple, purchased from Omega Engineering, and connected to a signal conditioner supplied by Measurement Computing Corporation (MCC), model USB-TC.

Shock speed is recorded by measuring the time intervals as the wave passes a series of six piezoelectric pressure transducers, Dynasen CA-1135. The spacing between pressure transducers is $150 \mathrm{~mm}$ for all three diagnostic sections, Figs. 2-4. The time intervals are recorded using a transducer readout module (TRM) designed and constructed by Argonne National Laboratory. The mounting hardware for the transducers are reproduced from the design and drawings of Ref. 15.

For the shocks presented in the present work, deceleration of the incident shock wave in the vicinity of the diagnostic is negligible, consistent with strong, ideal shocks. For the cyclohexene data, the maximum difference between an individual time interval and the average time was $3.1 \%$; for isobutyl nitrate, this discrepancy was $1.1 \%$. For the LS diagnostic, the laser is co-located with the fourth transducer port, and so the velocity at this position is well characterized.

The Dynasen pressure transducers have a response time less than $0.1 \mu \mathrm{s}$. For the experimental data presented in this work, the smallest average $d t$ between the pressure transducers is $153 \mu \mathrm{s}$. Based upon prior experience, ${ }^{1,2}$ the uncertainty in the shock velocity is estimated to be $\sim 0.2 \%$, based on the rise time of the pressure transducers, uncertainties in transducer location, and the data acquisition system, which results in an uncertainty in the temperature behind the incident shock, $T_{2}$, of $\sim 0.5 \%$. This degree of uncertainty is comparable to other similar apparatus.

Further details of the design, including technical drawings, are available from the authors on request.

\section{Laser-schlieren densitometry diagnostic}

The first diagnostic applied in the BST is laser-schlieren densitometry (LS). ${ }^{13,16,17}$ Laser-schlieren has the advantages of being simple, sensitive, highly resolved, general, and proportional. Over a short observation time of $\sim 10 \mu \mathrm{s}$, LS is able to measure unimolecular rate constants of up to $\sim 1 \times 10^{6} \mathrm{~s}^{-1}$. Furthermore, the technique allows for a probe of the overall kinetic mechanism by comparison of prediction to a global observable, density gradient. The diagnostic does not measure gas composition, and results are sensitive to perturbations in the flow (including the shock wave front). ${ }^{13}$ LS is also limited to low pressure due to deflection of the laser by turbulence in the boundary layer.

A continuous wave laser beam passes through the center of the driven section, perpendicular to the axis of the shock tube. An axial density gradient in the gas mixture causes a deflection in the path of the laser beam. The angle of deflection of the laser, $\theta$, is recorded using a differential detector and is converted to a density gradient, $d \rho / d x$, with knowledge of the refractivity of the gas mixture, $K_{L}$, and shock tube i.d., $W$.

The deflection angle recorded with the detector maps linearly to the density gradient in the shock tube ${ }^{13}$

$$
\theta=\frac{3}{2} K_{L} W \frac{d \rho}{d x}
$$

The density gradient is proportional to the total rate of heat release, $\Delta H$, in the driven gas mixture ${ }^{1}$

$$
\frac{d \rho}{d x}=\frac{\rho \sum_{j} r_{j}\left(\Delta H_{j}-\hat{C}_{p} T \Delta N_{j}\right)}{\rho_{0} u\left[\hat{C}_{p} T / \bar{M}-\hat{C}_{V} v^{2} / R\right]} .
$$

The summation occurs over $j$ reactions each occurring at rate $r$ with heat release $\Delta H$ and change in mole number $\Delta N$ at temperature $T$ and mixture heat capacity $\hat{C}_{p}$.

In the BST, instead of the helium-neon laser originally prescribed by Kiefer, ${ }^{13}$ a Fabrey-Perot red diode laser of similar wavelength (637 vs $633 \mathrm{~nm}$ ) has been employed, following the work of Annesley et al. ${ }^{18}$ The detector is a quadsegmented photodiode (UDT SPOT-9DMI) with electronics developed and constructed by Argonne National Laboratory.

The refractivity of the gas mixture, $K_{L}$, is found from a weighted average of the refractivity of individual species, $R_{L}$, where $X$ are mole fractions and $\bar{M}$ is the mixture average molecular weight ${ }^{13}$

$$
K_{L}=\sum_{i} R_{L_{i}} \frac{X_{i}}{\bar{M}}
$$

For the experiments conducted here, the values of $R_{L}$ in units of $\mathrm{cm}^{3} \mathrm{~mol}^{-1}$ are argon $=4.198,{ }^{19}$ krypton $=6.367,{ }^{19}$ cyclohexene $=27.3 \pm 0.3$, and isobutyl nitrite $=25.9 \pm 0.5$. Values of $R_{L}$ for cyclohexene and isobutyl nitrite are taken from the predictions of PhysChem module of ACD/Labs Percepta Platform software ${ }^{20}$ as published on ChemSpider. ${ }^{21}$ For comparison, Kiefer and Shah reported a value of $R_{L}=27.01$ for cyclohexene $e^{4}$ and Randazzo et al. reported $R_{L}=27.0$ for isobutyl nitrite. ${ }^{3}$ The differences in the values of $R_{L}$ for the reactant species between the original values and those used here vary by no more than $5 \%$. The contribution of the reactant $R_{L}$ to the overall value of $K_{L}$ is proportional to the mole fraction (2\%) and so the minor updates to the values are $R_{L}$ should have a negligible impact on the experimental density gradient.

\section{Mixture preparation}

Mixtures were prepared manometrically in a 721 glass vessel evacuated to below $1 \times 10^{-5}$ Torr. Mixtures were allowed to homogenize overnight before use. Argon, 99.999 9\%, and krypton, $99.999 \%$, were purchased from Corp Brothers. Cyclohexene, 99\%, Alfa Aesar, and isobutyl nitrite, 98\%, Frontier Scientific, were first degassed by repeated freeze-pump-thaw cycles using liquid nitrogen.

\section{RESULTS AND DISCUSSION}

\section{A. Pyrolysis of isobutyl nitrite}

Dissociation of isobutyl nitrite was previously reported by Randazzo et al. ${ }^{3}$ The reaction occurs at comparably low temperatures, due to the weak $\mathrm{O}-\mathrm{NO}$ bond $(\sim 41 \mathrm{kcal} / \mathrm{mol})$. The dissociation of isobutyl nitrite proceeds via steps $\mathrm{R} 1$ and $\mathrm{R} 2$ to form $\mathrm{NO}$, formaldehyde, and an isopropyl radical

$$
\begin{aligned}
\mathrm{iC}_{4} \mathrm{H}_{9} \mathrm{ONO} & \rightleftharpoons \mathrm{iC}_{4} \mathrm{H}_{9} \mathrm{O}+\mathrm{NO}, \\
\mathrm{iC}_{4} \mathrm{H}_{9} \mathrm{O} & \rightleftharpoons \mathrm{iC}_{3} \mathrm{H}_{7}+\mathrm{CH}_{2} \mathrm{O} .
\end{aligned}
$$


The rate of reaction $\mathrm{R} 2$ is much faster than reaction $\mathrm{R} 1 ;$ the early part of the LS experiments are essentially only sensitive to R1 allowing an accurate measure of its rate coefficient. At later times, the signal is dominated by exothermic secondary reactions, such as radical-radical recombination reactions. This transition from net
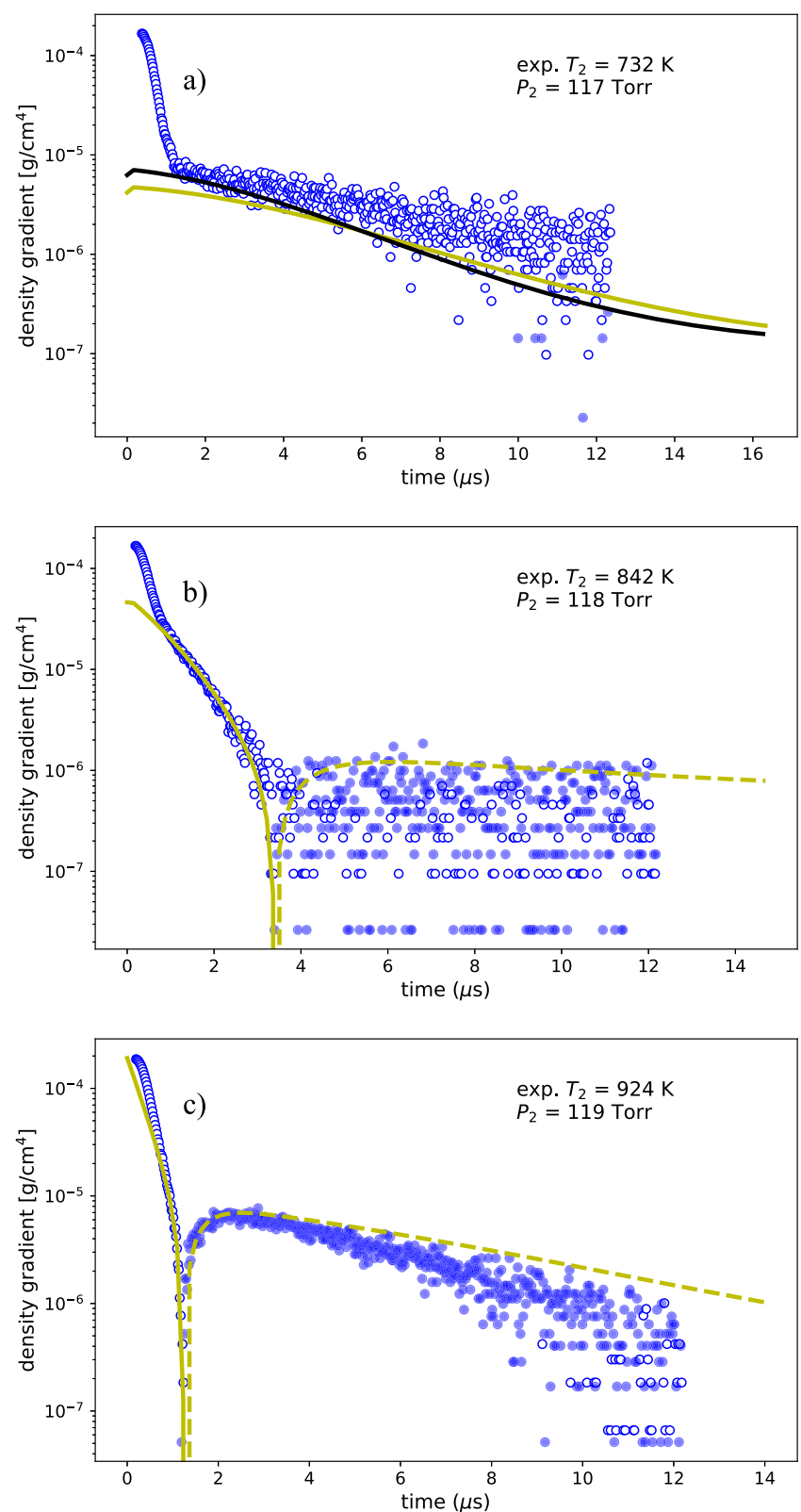

FIG. 7. $2 \%$ isobutyl nitrite dilute in Ar with model predictions. Open symbols and solid lines are positive density gradient; closed symbols and dashed lines are negative. Yellow (light gray in grayscale) lines represent predictions with unaltered published mechanisms. The black line in (a) shows the effect of a $50 \%$ increase in the rate of dissociation of isobutyl nitrite. The signals in (b) and (c) are representative of intermediate- and high-temperature shocks, respectively. endothermic to net exothermic reactions results in a sign change in the density gradient.

Twenty-two experiments were conducted in which isobutyl nitrite at $2 \%$ molar fraction in argon was shock heated to a nominal incident shock pressure, $P_{2}$, of 120 Torr in the temperature range $700 \mathrm{~K}<T_{2}<1000 \mathrm{~K}$. Sample LS plots are shown in Figs. 7(a)-7(c). Positive density gradients are represented by open circles, and negative density gradients are represented by filled circles. The detailed chemical mechanism of Randazzo et al. ${ }^{3}$ was used without alteration. The simulations were implemented in Cantera ${ }^{22}$ with thermodynamic data for all species except isobutyl nitrite taken from the Burcat database. ${ }^{23}$ The thermodynamic data for isobutyl nitrite was generated from group additivity estimates using the RMG website, rmg.mit.edu., ${ }^{24}$ consistent with the sources of thermodynamic data reported in Ref. 3. The yellow lines represent simulations using the mechanism of Randazzo et al. ${ }^{3}$ The downward spike in Figs. 7(b) and 7 (c) correspond to the time at which density gradient changes sign.

Rate coefficients for the initial dissociation of isobutyl nitrite were obtained from the density gradient at $t_{0}$. Small adjustments were made to $k_{1}$ to obtain the best fit between the mechanism and the current experiments. The resulting $k_{1}$ are represented by the circles in the Arrhenius plot in Fig. 8, and the recommended rate constant of Ref. 3 is the solid line. The agreement between the present work and the prior study of Randazzo et al. ${ }^{3}$ is excellent. The high degree of agreement confirms that the data produced by the BST do not contain any facility bias as compared with the diaphragmless shock tube at Argonne National Laboratory.

\section{B. Pyrolysis of cyclohexene}

Cyclohexene, $c-\mathrm{C}_{6} \mathrm{H}_{10}$, decomposition, previously studied by Kiefer and Shah, ${ }^{4}$ occurs at higher temperatures due to the activation barrier $(61.9 \mathrm{kcal} / \mathrm{mol})$ that has to be overcome. Twenty-eight experiments were conducted with mixtures of $2 \%$ molar fraction of cyclohexene in krypton, over the temperature range $1200 \mathrm{~K}<T_{2}$ $<1800 \mathrm{~K}$ at a nominal incident shock pressure of $P_{2}=120$ Torr. In this temperature range, cyclohexene undergoes a retro Diels-Alder

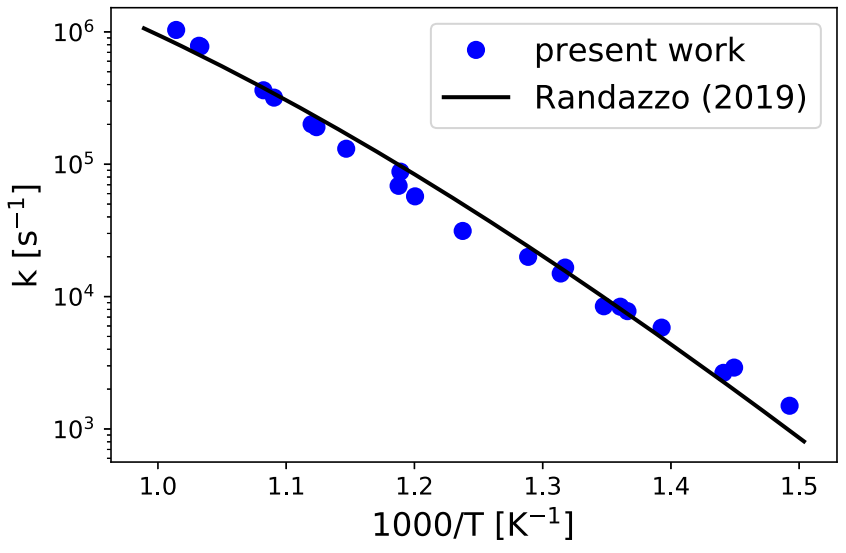

FIG. 8. Rate constants for the thermal decomposition of isobutyl nitrate. The symbols are the present work. The solid line is the literature value from Ref. 3 . 

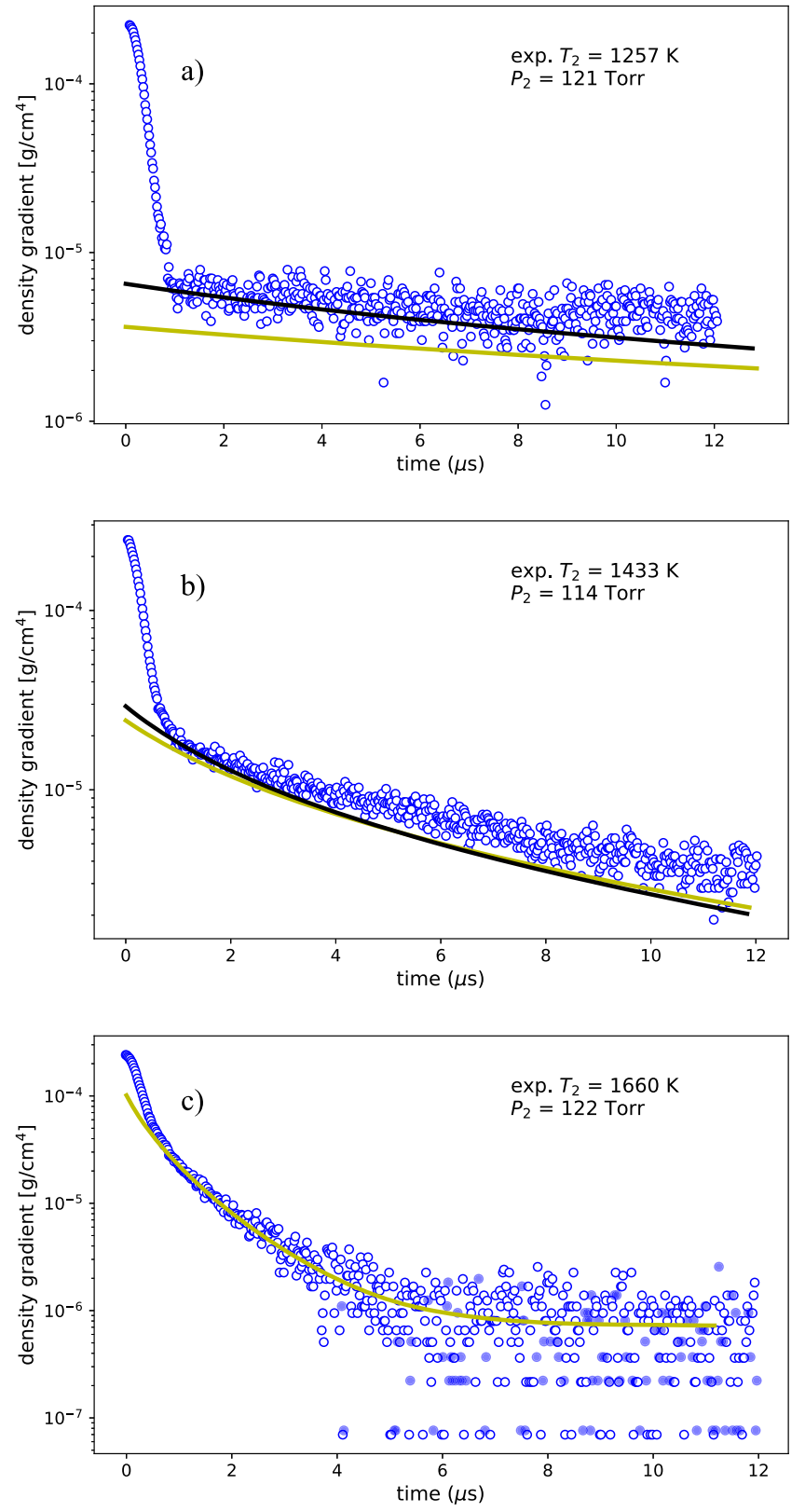

FIG. 9. 2\% cyclohexene dilute in $\mathrm{Kr}$ with model predictions. Open symbols and solid lines are positive density gradient; closed symbols and dashed lines are negative. Yellow (light gray in grayscale) lines represent predictions with unaltered published mechanisms. The black line in (a) shows the effect of a $80 \%$ increase in the rate of dissociation of cyclohexene; in (b) the black line represents an increase of $20 \%$. The nearly flat line in (a) $\left(T_{2}=1257 \mathrm{~K}\right)$ confirms that cyclohexene decomposition is nearly too slow to measure under these conditions.

ring opening to form ethene and 1,3-butadiene ${ }^{4}$

$$
\mathrm{cC}_{6} \mathrm{H}_{10} \rightleftharpoons \mathrm{C}_{2} \mathrm{H}_{4}+1,3 \mathrm{C}_{4} \mathrm{H}_{6} \text {. }
$$

It was suggested by Kiefer and Shah ${ }^{4}$ that below $1900 \mathrm{~K}$, the LS measurements could be modeled to experimental accuracy with

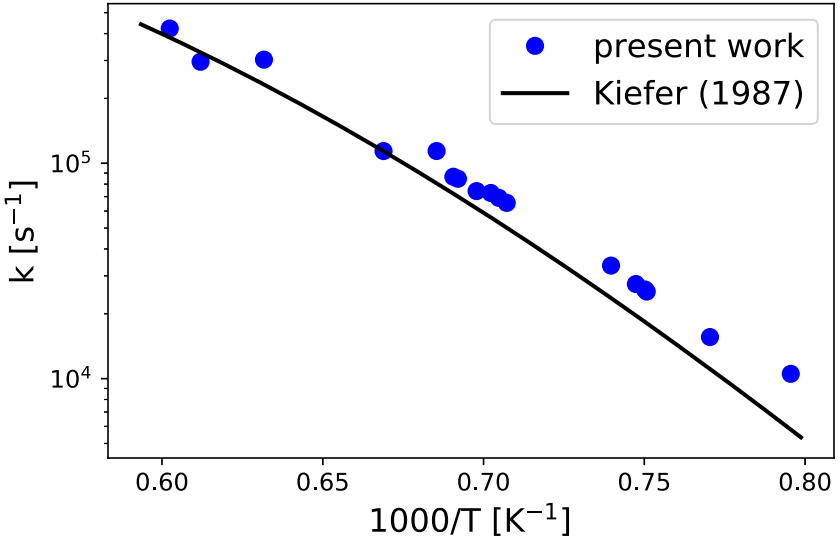

FIG. 10. Rate constants for the retro Diels-Alder of cyclohexene. The solid line is the literature value from Ref. 4.

only reaction, R3. However, for $T_{2} \gtrsim 1600 \mathrm{~K}$, dissociation of 1,3butadiene becomes significant, although the effect on the LS signals may remain negligible until higher temperatures when dissociation of cyclohexene is fast. Lockhart et al. ${ }^{25}$ recently published a LS study on the dissociation of 1,3-butadiene. This newer study provides a well-validated submechanism for the secondary chemistry of cyclohexene decomposition. This difference in the secondary chemistry model should not have a major impact on model agreement at early times when the density gradient is dominated by cyclohexene decomposition, but it will improve the model performance at longer times.

Sample laser schlieren plots are shown in Figs. 9(a)-9(c). The lines represent simulations using the combined mechanisms of Kiefer and Shah ${ }^{4}$ and Lockhart et al. ${ }^{25}$ As with the modeling of isobutyl nitrite, simulations were performed in Cantera; the thermodynamic data for all species were taken from Ref. 23. Rate coefficients for the initial dissociation of cyclohexene, $k_{3}$, were obtained from the density gradient at $t_{0}$. Figure 10 summarizes all the measured rate constants for $1200 \mathrm{~K}<T<1800 \mathrm{~K}$.

For cyclohexene, the current $k_{3}$ are consistently higher than the earlier work of Kiefer and Shah. ${ }^{4}$ However, the results are within $10 \%-50 \%$ of Kiefer's RRKM model, except for the lowest $\mathrm{T}$ point which was within $80 \%$. Considering the differences between the shock tubes, data acquisition systems, thermochemical properties, and secondary chemistry mechanisms, the agreement with Kiefer's work is within expected uncertainties.

\section{CONCLUSIONS}

A new diaphragmless shock tube with software control and actuation of valving is presented. The instrument is presently configured for low-pressure experiments utilizing laser-schlieren densitometry. Instrument design includes options for easy reconfiguration to perform laser-absorption measurements and ignition delay studies.

Repetition of studies on the pyrolysis of both isobutyl nitrite and cyclohexene shows that the BST and laser-schlieren diagnostic system are able to reproduce published results to within experimental uncertainty. 


\section{ACKNOWLEDGMENTS}

This research was funded by Brown University and the National Science Foundation through Award No. CBET-1553366, with Dr. Song-Charng Kong as the program manager. R.S.T. was support by the U.S. Department of Energy, Office of Basic Energy Sciences, Division of Chemical Sciences, Geosciences, and Biosciences through Argonne National Laboratory, Contract No. DEAC02-06CH11357. We are grateful to the High Energy Physics group at Argonne National Laboratory for construction of the TRM and photodiode electronics. M.E.F. thanks Aaron Rosenthal for assistance with the construction of the shock tube facility and Kayli Sarpu for continuing shock tube control software development.

\section{REFERENCES}

${ }^{1}$ R. S. Tranter and B. R. Giri, Rev. Sci. Instrum. 79, 094103 (2008).

${ }^{2}$ J. B. Randazzo and R. S. Tranter, Rev. Sci. Instrum. 86, 016117 (2015).

${ }^{3}$ J. B. Randazzo, M. E. Fuller, C. F. Goldsmith, and R. S. Tranter, Proc. Combust. Inst. 37, 703 (2019).

${ }^{4}$ J. H. Kiefer and J. N. Shah, J. Phys. Chem. 91, 3024 (1987).

${ }^{5}$ R. K. Hanson and D. F. Davidson, Prog. Energy Combust. Sci. 44, 103 (2014).

${ }^{6}$ R. S. Tranter, K. Brezinsky, and D. Fulle, Rev. Sci. Instrum. 72, 3046 (2001)

${ }^{7}$ American Society of Mechanical Engineers, ASME Boiler and Pressure Vessel Code Division 1 (ASME, 2013).

${ }^{8}$ American Society of Mechanical Engineers, in ASME Boiler and Pressure Vessel Code Division 1 (ASME, 2013), Chap. Y, p. 630.
${ }^{9}$ F. Kirkemo, Analysis of Bolted Joints (ASME, 2002), PVP2002-1087.

${ }^{10}$ E. Oberg, F. D. Jones, H. L. Horton, and H. H. Ryffel, in Machinery's Handbook, 29th ed., edited by C. J. McCauley (Industrial Press, 2012).

${ }^{11}$ P. R. Yoder, Mounting Optics in Optical Instruments (SPIE, 2008).

${ }^{12}$ See www.solidworks.com for Dassault Systémes, Solidworks.

${ }^{13}$ J. H. Kiefer, in Shock Waves in Chemistry, edited by A. Lifshitz (Marcel Dekker, Inc., 1981), Chap. 5, pp. 219-277.

${ }^{14}$ C. J. E. Aul, “An experimental study into the ignition of methane and ethane blends in a new shock-tube facility," Master's thesis, Texas A\&M University, 2009.

${ }^{15}$ R. S. Tranter and P. T. Lynch, Rev. Sci. Instrum. 84, 094102 (2013).

${ }^{16}$ J. H. Kiefer and R. W. Lutz, J. Chem. Phys. 44, 658 (1966).

${ }^{17}$ J. H. Kiefer and R. W. Lutz, J. Chem. Phys. 44, 668 (1966).

${ }^{18}$ C. J. Annesley, J. B. Randazzo, S. J. Klippenstein, L. B. Harding, A. W. Jasper, Y. Georgievskii, B. Ruscic, and R. S. Tranter, J. Phys. Chem. A 119, 7872 (2015).

${ }^{19}$ W. Gardiner, Y. Hidaka, and T. Tanzawa, Combust. Flame 40, 213 (1981).

${ }^{20}$ See www.acdlabs.com/products/percepta/predictors.php for ACD/Labs, Percepta platform.

${ }^{21}$ See www.chemspider.com/ for Royal Society of Chemistry, Chemspider.

${ }^{22}$ D. G. Goodwin, H. K. Moffat, and R. L. Speth, "Cantera: An object-oriented software toolkit for chemical kinetics, thermodynamics, and transport processes," version 2.2.1, 2016

${ }^{23}$ E. Goos, A. Burcat, and B. Ruscic, "Ideal gas thermodynamic data in polynomial form for combustion and air pollution use," available at http://garfield.chem.elte.hu/Burcat/burcat.html.

${ }^{24}$ C. W. Gao, J. W. Allen, W. H. Green, and R. H. West, Comput. Phys. Commun. 203, 212 (2016).

${ }^{25}$ J. P. A. Lockhart, C. F. Goldsmith, J. B. Randazzo, B. Ruscic, and R. S. Tranter, J. Phys. Chem. A 121, 3827 (2017). 